\title{
Fusion hindrance and Pauli blocking in ${ }^{58} \mathrm{Ni}+{ }^{64} \mathrm{Ni}$
}

\author{
A. M. Stefanini $\odot,{ }^{1}$ G. Montagnoli, ${ }^{2}$ M. Del Fabbro, ${ }^{2}$ G. Colucci, ${ }^{2}$ P. Čolović $\odot,{ }^{3}$ L. Corradi,,${ }^{1}$ E. Fioretto, ${ }^{1}$ F. Galtarossa, ${ }^{1}$ \\ A. Goasduff $\odot,{ }^{2}$ J. Grebosz $\odot,{ }^{4}$ M. Heine $\odot,{ }^{5}$ G. Jaworski, ${ }^{6}$ M. Mazzocco $\odot,{ }^{2}$ T. Mijatovic, ${ }^{3}$ S. Szilner, ${ }^{3}$ M. Bajzek, ${ }^{3}$ \\ D. Brugnara, ${ }^{1}$ M. Siciliano ${ }^{(1)}{ }^{1}$ and I. Zanon ${ }^{7,1}$ \\ ${ }^{1}$ INFN, Laboratori Nazionali di Legnaro, I-35020 Legnaro (Padova), Italy \\ ${ }^{2}$ Dipartimento di Fisica e Astronomia, Università di Padova, and INFN-Padova, I-35131 Padova, Italy \\ ${ }^{3}$ Ruđer Bošković Institute, HR-10002 Zagreb, Croatia \\ ${ }^{4}$ Institute of Nuclear Physics, Polish Academy of Sciences, PL 31-342 Cracow, Poland \\ ${ }^{5}$ IPHC, CNRS-IN2P3, Université de Strasbourg, F-67037 Strasbourg Cedex 2, France \\ ${ }^{6}$ Heavy Ion Laboratory, Univ. of Warsaw, Warsaw, Poland \\ ${ }^{7}$ Dipartimento di Fisica e Scienze della Terra, Università of Ferrara, I-44121 Ferrara, Italy
}

(Received 27 August 2019; published 31 October 2019)

\begin{abstract}
Background: ${ }^{58} \mathrm{Ni}+{ }^{64} \mathrm{Ni}$ is the first case where the influence of positive $Q$-value transfer channels on subbarrier fusion was evinced, in a very well known experiment by Beckerman et al. [Phys. Rev. Lett. 45, 1472 (1980)], by comparing with the two systems ${ }^{58} \mathrm{Ni}+{ }^{58} \mathrm{Ni}$ and ${ }^{64} \mathrm{Ni}+{ }^{64} \mathrm{Ni}$. Subsequent measurements on ${ }^{64} \mathrm{Ni}+{ }^{64} \mathrm{Ni}$ showed that fusion hindrance is clearly present in this case. On the other hand, no indication of hindrance can be observed for ${ }^{58} \mathrm{Ni}+{ }^{64} \mathrm{Ni}$ down to the measured level of $0.1 \mathrm{mb}$.

Purpose: Measuring deep subbarrier fusion cross sections for ${ }^{58} \mathrm{Ni}+{ }^{64} \mathrm{Ni}$, where the influence of positive $Q$ value transfer channels on near-barrier fusion was evidenced previously, in order to investigate whether hindrance shows up.

Methods: $167-200 \mathrm{MeV}{ }^{58} \mathrm{Ni}$ beams from the XTU Tandem Accelerator of INFN-Laboratori Nazionali di Legnaro were used, bombarding thin metallic ${ }^{64} \mathrm{Ni}\left(50 \mu \mathrm{g} / \mathrm{cm}^{2}\right)$ enriched to $99.6 \%$ in mass 64 . An electrostatic beam deflector allowed fusion evaporation residues to be detected at very forward angles in a detector telescope. Results: The excitation function has been extended by two orders of magnitude downward. The cross sections for ${ }^{58} \mathrm{Ni}+{ }^{64} \mathrm{Ni}$ continue decreasing very smoothly below the barrier, down to $\simeq 1 \mu \mathrm{b}$. The logarithmic slope of the excitation function increases slowly, showing a tendency to saturate at the lowest energies. No maximum of the astrophysical $S$ factor is observed. Coupled-channel (CC) calculations using a Woods-Saxon potential and including inelastic excitations only underestimate the subbarrier cross sections by a large amount. Good agreement is found by adding two-nucleon transfer couplings to a schematical level. This behavior is quite different from what already observed for ${ }^{64} \mathrm{Ni}+{ }^{64} \mathrm{Ni}$ (no positive $Q$-value transfer channels available), where a clear low-energy maximum of the $S$ factor appears, and whose excitation function is overestimated by a standard Woods-Saxon CC calculation.

Conclusions: No hindrance effect is observed in ${ }^{58} \mathrm{Ni}+{ }^{64} \mathrm{Ni}$ in the measured energy range. This trend at deep subbarrier energies reinforces the recent suggestion that the availability of several states following transfer with $Q>0$ effectively counterbalances the Pauli repulsion that, in general, is predicted to reduce tunneling probability inside the Coulomb barrier.
\end{abstract}

DOI: 10.1103/PhysRevC.100.044619

\section{INTRODUCTION}

The sequence of stable nickel isotopes from ${ }^{58} \mathrm{Ni}$ to ${ }^{64} \mathrm{Ni}$ offers several opportunities of studying fusion dynamics near and below the Coulomb barrier. The early experiments on fusion of $\mathrm{Ni}+\mathrm{Ni}$ systems [1] are well known and indicated for the first time the possible influence of transfer reactions on near- and subbarrier cross sections. The excitation functions of the three systems ${ }^{58} \mathrm{Ni}+{ }^{58} \mathrm{Ni},{ }^{58} \mathrm{Ni}+{ }^{64} \mathrm{Ni}$, and ${ }^{64} \mathrm{Ni}+{ }^{64} \mathrm{Ni}$, besides the trivial differences due to the varying Coulomb barriers, show a remarkable feature; that is, the contrasting slope of the asymmetric system ${ }^{58} \mathrm{Ni}+{ }^{64} \mathrm{Ni}$, when compared with the other two symmetric cases. Indeed, the cross sections of ${ }^{58} \mathrm{Ni}+{ }^{64} \mathrm{Ni}$ decrease much slower with decreasing energy. Shortly after, this was associated [2] with the availability, only in this system, of neutron-transfer channels with positive $Q$ values. Later experiments for ${ }^{58} \mathrm{Ni}+{ }^{64} \mathrm{Ni}$ [3] confirmed the flat shape of the excitation function, but the measured cross sections were anyway limited to $\sigma \geqslant 0.1 \mathrm{mb}$.

In more recent years it was found for many systems [4] that, at deep subbarrier energies, the cross section decreases very rapidly [5], so that the excitation function is much steeper than the prediction of standard coupled-channels (CC) calculations. This phenomenon was named fusion hindrance. One of the first systems where this effect was clearly identified is ${ }^{64} \mathrm{Ni}+{ }^{64} \mathrm{Ni}$ [6]. The original data of Beckerman et al. [1] were extended down to the level of $\approx 25 \mathrm{nb}$ and the threshold 
of hindrance is around $0.1 \mathrm{mb}$. The effect was recognized also in the case of ${ }^{58} \mathrm{Ni}+{ }^{58} \mathrm{Ni}$ at the level of $0.05 \mathrm{mb}$ from the data of Ref. [1].

Low-energy hindrance is a matter of continuing experimental and theoretical interest. In the sudden approach proposed by Misicu and Esbensen [7,8], a double-folding potential is adopted (M3Y + repulsion), producing a shallow pocket as a consequence of the incompressibility of nuclear matter. This CC model has been quite successful in reproducing the hindrance behavior in a number of cases [9].

Alternatively, Ichikawa et al. [10] proposed an adiabatic neck formation between the colliding nuclei in the overlap region, leading to hindrance. More recently, Simenel et al. [11] introduced a new microscopic model and demonstrated, on the basis of density-constrained frozen Hartree-Fock calculations, that the main effect of Pauli repulsion is to reduce tunneling probability inside the Coulomb barrier. It has been pointed out as well that, when positive $Q$-value transfer channels are available to the system, this effect of Pauli blocking may be reduced or disappear altogether [12], because several final states can be populated, and valence nucleons can flow between the two nuclei, thus initiating fusion. This corresponds to what was observed for the system ${ }^{40} \mathrm{Ca}+{ }^{96} \mathrm{Zr}$ that was investigated a few years ago $[13,14]$. The flat shape of its subbarrier fusion excitation function is very peculiar and was suggested [15] to originate from the couplings to several $Q>0$ nucleon (neutron and proton) transfer channels. ${ }^{40} \mathrm{Ca}+{ }^{96} \mathrm{Zr}$ was studied down to very small cross sections $(2 \mu \mathrm{b})$ and the phenomenon of fusion hindrance does not show up.

If that interpretation is correct, we expect a similar behavior for ${ }^{58} \mathrm{Ni}+{ }^{64} \mathrm{Ni}$, which is a very attractive case in this sense because the $Q$ values for the neutron transfer channels are $+3.9 \mathrm{MeV}$, for $2 n$ and $4 n$ pick up, and where a clear evidence of transfer couplings was already indicated below the barrier. The appearance of fusion hindrance in this case, would put serious doubts on the suggestion that Pauli blocking is not effective (or weakened) in systems with $Q>0$ transfer channels.

The experiment was performed very recently, so that the low-energy part of the excitation function for ${ }^{58} \mathrm{Ni}+{ }^{64} \mathrm{Ni}$ has been extended down by about two orders of magnitude. This article reports on the results of these measurements. Section II describes the experimental setup in some detail and shows the results obtained. Coupled-channel calculations will be presented in Sec. III. A comparison of ${ }^{58} \mathrm{Ni}+{ }^{64} \mathrm{Ni}$ fusion with the behavior of other ${ }^{64} \mathrm{Ni}+{ }^{64} \mathrm{Ni}$ and ${ }^{40} \mathrm{Ca}+{ }^{96} \mathrm{Zr}$ systems will be performed in Sec. IV. The conclusions of the present work are summarized in Sec. V.

\section{EXPERIMENTAL SETUP AND RESULTS}

Fusion-evaporation cross sections have been measured for the system ${ }^{58} \mathrm{Ni}+{ }^{64} \mathrm{Ni}$ at several energies near and below the Coulomb barrier, using the ${ }^{58} \mathrm{Ni}$ beam provided by the XTU Tandem Accelerator of the Laboratori Nazionali di Legnaro (LNL) of INFN in the energy range 167-201 MeV. The beam intensity was 3-4 pnA. The targets were $50 \mu \mathrm{g} / \mathrm{cm}^{2}$ nickel evaporations on $15 \mu \mathrm{g} / \mathrm{cm}^{2}$ carbon backings, with an enrichment of $99.6 \%$ in mass 64 . Since ${ }^{64} \mathrm{Ni}$ is the heaviest stable nickel isotope, the correction introduced in the crosssection determination by the lighter isotopes was very small or negligible. As for possible target contaminants with $\mathrm{Z}<$ 28 , the mass of the corresponding compound nuclei is very different, so that the fusion-evaporation residues would have been easily separated out by the combined energy-time-offlight measurements described below. The target and backing thicknesses were taken into account when determining an effective beam energy at the center of the ${ }^{64} \mathrm{Ni}$ target. The beam energy of the XTU Tandem is determined to better than $1 / 800$ on an absolute scale, i.e., about $250 \mathrm{keV}$ at $200 \mathrm{MeV}$. The energy was varied only downwards to minimize hysteresis effects in the energy-defining $90^{\circ}$ magnet. At each energy, the beam was focused to the center of the target by using a fluorescent quartz.

Fusion-fission is negligible for ${ }^{58} \mathrm{Ni}+{ }^{64} \mathrm{Ni}$ in the measured energy range; hence fusion cross sections were obtained by detecting at forward angles the evaporation residues (ERs) following compound nucleus formation. The ERs were separated from the beam by using the electrostatic deflector (see Ref. [16] and references therein) usually employed for fusion measurements at LNL.

Following beam separation, the ERs were detected by two microchannel-plate detectors (MCP) $30 \times 50 \mathrm{~mm}^{2}$, a transverse-field ionization chamber (IC), and, finally, a $600 \mathrm{~mm}^{2}$ silicon detector placed in the same gas $\left(\mathrm{CH}_{4}\right)$ volume. This sequence makes up a $\Delta E-E$-TOF telescope, where the silicon detector provides the residual energy $E$ and the start signal used for the two independent time-of-flights (TOFs), and for triggering the data acquisition.

Four silicon detectors were installed to normalize between the different runs, by measuring the Rutherford scattering from the target and for monitoring the beam position and focus conditions. They were placed around $300 \mathrm{~mm}$ from the target at the same scattering angle $\theta_{\mathrm{lab}}=16^{\circ}$, above and below, and to the left and right of the beam direction.

The absolute cross-section scale was fixed by equalizing the ER yield we obtained at the highest measured energy $\left(E_{\text {lab }}=198.8 \mathrm{MeV}\right)$ to the corresponding cross section quoted in Ref. [1]. Statistical errors are $\sim 2 \%$ near the barrier and become much larger at low energies where only few fusion events were detected.

The set of fusion cross sections we have obtained for ${ }^{58} \mathrm{Ni}+{ }^{64} \mathrm{Ni}$ is reported in Table I and shown in Fig. 1 with full blue dots, together with the previous results for the same system and for ${ }^{58} \mathrm{Ni}+{ }^{58} \mathrm{Ni}$ [1] (black crosses and open diamonds, respectively), and with the more recent measurements of Jiang et al. [6] on ${ }^{64} \mathrm{Ni}+{ }^{64} \mathrm{Ni}$ (red triangles). It is evident that the cross sections for ${ }^{58} \mathrm{Ni}+{ }^{64} \mathrm{Ni}$ continue decreasing very smoothly below the barrier, while the two symmetric systems have much steeper excitation functions. This trend is clearly observed down to the lowest-measured cross section of $\simeq 1.3 \mu \mathrm{b}$.

Figure 2 shows the logarithmic slope of the excitation function, derived from the measured cross sections, as the incremental ratio of two nearby points, for the various $\mathrm{Ni}+\mathrm{Ni}$ systems. In this case, the existing data on ${ }^{58} \mathrm{Ni}+{ }^{60} \mathrm{Ni}$ [17] have been added to the systematics. It appears that the trend of this system is similar to that of ${ }^{58} \mathrm{Ni}+{ }^{64} \mathrm{Ni}$. For both 
TABLE I. Fusion cross sections measured in this work for ${ }^{58} \mathrm{Ni}+$ ${ }^{64} \mathrm{Ni}$ (see text).

\begin{tabular}{lcc}
\hline \hline$E_{\text {c.m. }}(\mathrm{MeV})$ & $\sigma(\mathrm{mb})$ & Error $(\mathrm{mb})$ \\
\hline 86.92 & 0.00132 & 0.00093 \\
87.44 & 0.00325 & 0.00098 \\
87.97 & 0.0103 & 0.0035 \\
88.50 & 0.0280 & 0.0100 \\
89.02 & 0.0585 & 0.0127 \\
89.76 & 0.122 & 0.018 \\
90.75 & 0.774 & 0.053 \\
92.28 & 3.50 & 0.096 \\
94.59 & 15.56 & 0.23 \\
99.26 & 75.28 & 1.41 \\
104.31 & 166.0 & 2.3 \\
\hline \hline
\end{tabular}

these cases, in the measured energy range, the slope increases slowly below the barrier with decreasing energy without notable irregularities. It does not reach the value expected for a constant astrophysical $S$ factor $\left(L_{C S}\right.$ in Fig. 2); rather, it seems to saturate around $2 \mathrm{MeV}^{-1}$. Indeed, the influence of $2 n$-transfer couplings at the lowest energies was qualitatively suggested also for ${ }^{58} \mathrm{Ni}+{ }^{60} \mathrm{Ni}$ (shown in the figure). For the other two symmetric systems, Fig. 2 shows (as already known) that the slope clearly overcomes the $L_{C S}$ value, thus presenting hindrance.

The CC calculations we are going to present in the next section and the comparison with ${ }^{64} \mathrm{Ni}+{ }^{64} \mathrm{Ni}$ will tell us more about this point.

\section{COUPLED-CHANNEL ANALYSIS}

The CCFULL code [18] has been used to perform CC calculations for ${ }^{58} \mathrm{Ni}+{ }^{64} \mathrm{Ni}$. The ion-ion potential was a Woods Saxon parametrization with well depth $V_{0}=151.85$ $\mathrm{MeV}$, diffuseness $a=0.67 \mathrm{fm}$, and radius parameter $r_{0}=$ $1.10 \mathrm{fm} . V_{0}$ is much deeper than what one obtains from the Akyüz-Winther potential [19]. It is used to remove unwanted

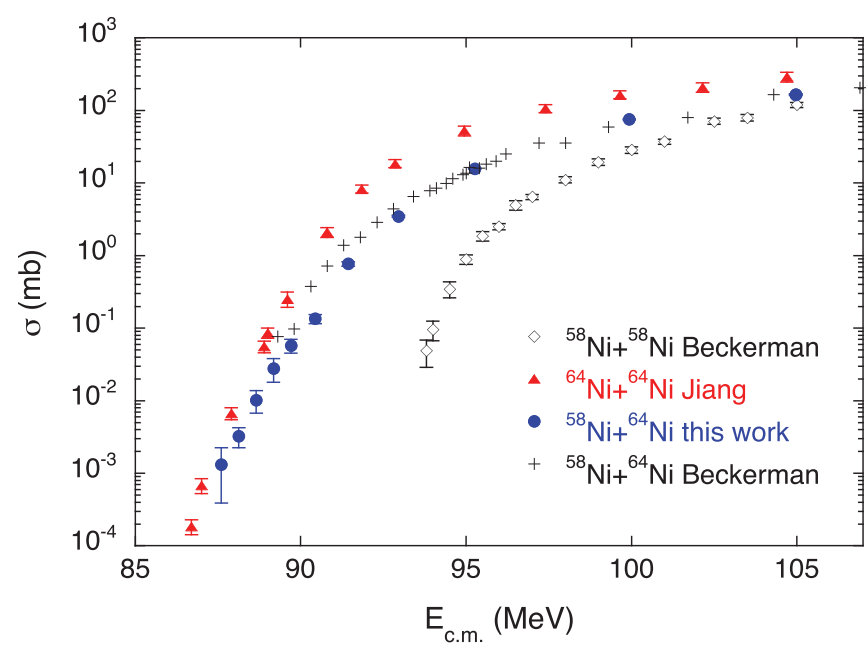

FIG. 1. Fusion excitation functions for several Ni $+\mathrm{Ni}$ systems from the present and previous measurements. The data marked Beckerman and Jiang are from Refs. [1,6].

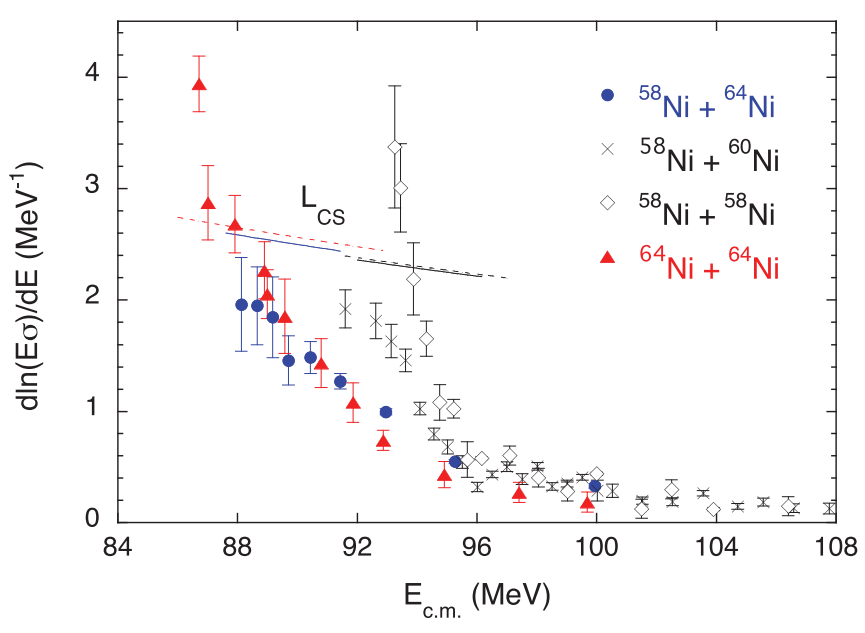

FIG. 2. Logarithmic derivatives for several $\mathrm{Ni}+\mathrm{Ni}$ systems. The $L_{C S}$ curves for ${ }^{58} \mathrm{Ni}+{ }^{64} \mathrm{Ni}$ and ${ }^{64} \mathrm{Ni}+{ }^{64} \mathrm{Ni}$ appear more on the left (full line and dashed line, respectively). The curves for ${ }^{58} \mathrm{Ni}+{ }^{58} \mathrm{Ni}$ and ${ }^{58} \mathrm{Ni}+{ }^{60} \mathrm{Ni}$ appear more on the right (full line and dashed line, respectively).

oscillations of the low-energy excitation function that appear if the potential is too shallow, and consequently the incomingwave boundary condition is not correctly applied. The parameters have been chosen to obtain a good data fit in the barrier region $\sigma=10-100 \mathrm{mb}$, when all channels, including the two-nucleon transfer (see later on) are taken into account in the calculations.

The nuclear-structure information of the low-lying collective modes of ${ }^{58} \mathrm{Ni}$ and ${ }^{64} \mathrm{Ni}$ is reported in Table II. The two nickel isotopes have quadrupole states at similar excitation energies and strengths. In the calculations we have considered up to two quadrupole phonons and only one octupole phonon (which has much higher excitation energy) in both nuclei.

Figure 3 shows the results of the calculations when compared with the present experimental data (full dots). We notice that the $\mathrm{CC}$ results (upper dashed line) strongly underestimate the data below the barrier. This indicates the possible effect of transfer couplings. Indeed, in the recent work on ${ }^{40} \mathrm{Ca}+$ ${ }^{96} \mathrm{Zr}$ [15], it was pointed out that the effective $Q$ values for two-neutron as well as two-proton transfer are positive and both transfer channels can therefore influence the fusion. The situation is the same for ${ }^{58} \mathrm{Ni}+{ }^{64} \mathrm{Ni}$ where the corresponding $Q$ values are +3.89 and $+2.6 \mathrm{MeV}$.

TABLE II. Excitation energies $E_{x}$, spin and parities $\lambda^{\pi}$, reduced transition probabilities and deformation parameters $\beta_{\lambda}$ [22,23] for the lowest quadrupole and octupole modes of ${ }^{58} \mathrm{Ni}$ and ${ }^{64} \mathrm{Ni}$. The $\mathrm{B}(E 3)$ value for ${ }^{64} \mathrm{Ni}$ was taken from Ref. [24]. The radius parameter used to estimate the $\beta_{\lambda}$ values is $1.20 \mathrm{fm}$. Nuclear and Coulomb deformation parameters have been taken to be the same in the present $\mathrm{CC}$ analysis.

\begin{tabular}{lcccl}
\hline \hline Nucleus & $E_{x}(\mathrm{MeV})$ & $\lambda^{\pi}$ & $\mathrm{B}(E \lambda) \downarrow($ W.u. $)$ & \multicolumn{1}{c}{$\beta_{\lambda}$} \\
\hline${ }^{58} \mathrm{Ni}$ & 1.454 & $2^{+}$ & $10.0(3)$ & 0.183 \\
& 4.475 & $3^{-}$ & $12.6(12)$ & 0.20 \\
${ }^{64} \mathrm{Ni}$ & 1.346 & $2^{+}$ & $9.6(1)$ & 0.179 \\
& 3.560 & $3^{-}$ & $13.2(26)$ & $0.203)$ \\
\hline \hline
\end{tabular}




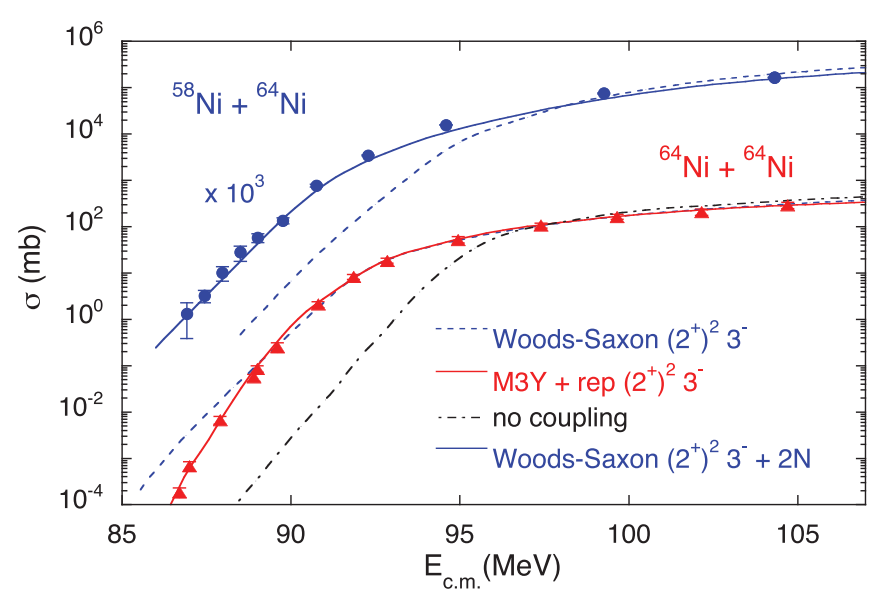

FIG. 3. Fusion excitation function of ${ }^{58} \mathrm{Ni}+{ }^{64} \mathrm{Ni}$ (full dots) and ${ }^{64} \mathrm{Ni}+{ }^{64} \mathrm{Ni}$ (triangles), compared with $\mathrm{CC}$ calculation (see text).

Therefore, further calculations were performed, including a two-nucleon transfer channel $(+2 N)$ besides the collective surface modes discussed above, using the approximate treatment of CCFULL where a pair transfer coupling between the ground states may be included. This uses the macroscopic coupling form factor given in Ref. [20]. The coupling strength $F_{t}=0.6 \mathrm{MeV}$ has been used, adjusted for the best data fit. This rather large strength may be explained by the fact that it includes both proton and neutron transfer channels and the upper full line shows the result in Fig. 3.

In the $S$-factor representation (Fig. 4) we can reach analogous conclusions. Indeed, only including in the $\mathrm{CC}$ calculations the transfer coupling we have been able to reproduce the experimental $S$-factor trend where no maximum has been observed vs energy.

The present results, and the comparison to $\mathrm{CC}$ calculations, indicate that hindrance does not show up in ${ }^{58} \mathrm{Ni}+{ }^{64} \mathrm{Ni}$ in

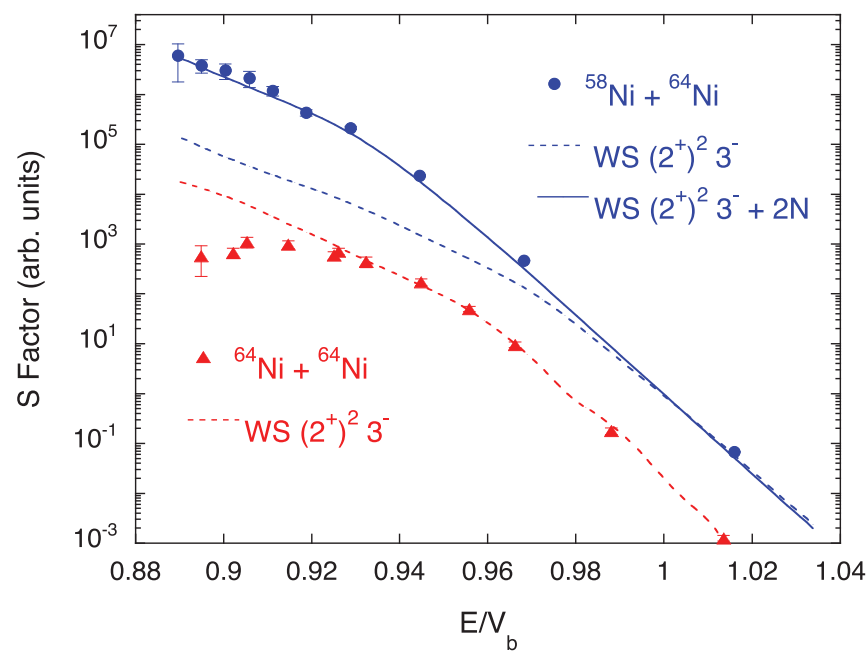

FIG. 4. $S$ factor for ${ }^{58,64} \mathrm{Ni}+{ }^{64} \mathrm{Ni}$ compared with $\mathrm{CC}$ calculations by using a WS potential (upper and lower dashed line, respectively). The full line is the result of a $\mathrm{CC}$ calculation for ${ }^{58} \mathrm{Ni}+{ }^{64} \mathrm{Ni}$ including the additional coupling to the two-nucleon transfer channel. the measured energy range. At lower energies, we know that hindrance must appear, because the $Q$ value for fusion is negative $Q_{\text {fus }}=-52.7 \mathrm{MeV}$ [21].

\section{COMPARISON WITH ${ }^{64} \mathrm{Ni}+{ }^{64} \mathrm{Ni}$ AND ${ }^{40} \mathrm{Ca}+{ }^{96} \mathrm{Zr}$ SYSTEMS}

A comparison with the nearby system ${ }^{64} \mathrm{Ni}+{ }^{64} \mathrm{Ni}$ is significant because, in this case, the hindrance phenomenon is present [6]. CCFULL calculations have been performed by using the structure information of Table II, and the WoodsSaxon ion-ion potential with parameters $V_{0}=75.98 \mathrm{MeV}$, diffuseness $a=0.676 \mathrm{fm}$, and radius parameter $r_{0}=1.202 \mathrm{fm}$, as quoted in the original article [6]. In analogy with ${ }^{58} \mathrm{Ni}+$ ${ }^{64} \mathrm{Ni}$, couplings to two quadrupole phonons and one octupole phonon have been considered. The result of the CC calculation is reported in Fig. 3 (see also Ref. [4]). We notice immediately that, at variance with ${ }^{58} \mathrm{Ni}+{ }^{64} \mathrm{Ni}$, the measured cross sections drop below the calculation (lower dashed line) at low energies. Only by using a shallow M3Y + repulsion potential $[7,8]$ (lower full line) does one get a good data fit, as already known.

The comparison of the excitation functions for the two systems with the corresponding CC calculations confirms that they behave quite differently in the low-energy region. This is even more clear in the astrophysical $S$-factor representation, as reported in Fig. 4. The experimental $S$ factors of the two systems are found on opposite sides with respect to the corresponding calculations using standard WS potentials. In particular, the maximum of $S$ observed for ${ }^{64} \mathrm{Ni}+{ }^{64} \mathrm{Ni}$ but not for ${ }^{58} \mathrm{Ni}+{ }^{64} \mathrm{Ni}$, is not reproduced by the $\mathrm{CC}$ calculations.

The case of ${ }^{58} \mathrm{Ni}+{ }^{64} \mathrm{Ni}$, on the other hand, is very similar to ${ }^{40} \mathrm{Ca}+{ }^{96} \mathrm{Zr}[13,14]$ because of the flat shape of the two subbarrier fusion excitation functions, probably originating in both cases from the couplings to several $Q>0$ nucleon transfer channels.

In ${ }^{40} \mathrm{Ca}+{ }^{96} \mathrm{Zr}$, fusion hindrance does not show up and this rather unusual behavior is also due to the $Q>0$ transfer couplings. Indeed, the barrier distribution of this system displays a very long tail towards low energies [14], and we know the hindrance phenomenon shows up below the energy where the barrier distribution vanishes. This led to the suggestion [12] that the reaction mechanism involves the Pauli exclusion principle that in general produces fusion hindrance [11], apart from the cases, as mentioned in the introduction, where several final states can be populated by nucleon transfer with $Q>0$.

The absence of hindrance in the present case of ${ }^{58} \mathrm{Ni}+$ ${ }^{64} \mathrm{Ni}$ reinforces that suggestion. The strong dissimilarity with respect to the nearby case of ${ }^{64} \mathrm{Ni}+{ }^{64} \mathrm{Ni}$ contributes to clarify the subbarrier fusion dynamics and prompts us to contemplate the possibility that the repulsive part of the potential in the Misicu-Esbensen model [7,8] is actually a consequence of the Pauli exclusion principle.

\section{SUMMARY AND CONCLUSIONS}

In this paper we have presented the results of fusion excitation function measurements for the system ${ }^{58} \mathrm{Ni}+{ }^{64} \mathrm{Ni}$. The 
experiment was performed by using the ${ }^{58} \mathrm{Ni}$ beam of the XTU Tandem Accelerator of the LNL, and the excitation function obtained previously $[1,3]$ has been extended downwards by two orders of magnitude to about $1 \mu \mathrm{b}$. We observe that the logarithmic slope of the excitation function has a slow increase and tends to saturate at the lowest energies. The astrophysical $S$ factor does not show any maximum vs energy.

$\mathrm{CC}$ calculations using a Woods-Saxon potential have been performed. The results underestimate the subbarrier cross sections by a large amount, when only inelastic excitations are included. Good agreement is, however, found by schematically adding the coupling to the two-nucleon transfer. Both two-neutron and two-proton transfer have positive effective ground state $Q$ values. The behavior of ${ }^{58} \mathrm{Ni}+{ }^{64} \mathrm{Ni}$ is quite different from what already observed for ${ }^{64} \mathrm{Ni}+{ }^{64} \mathrm{Ni}$, where no positive $Q$-value transfer channels are available. In this case a clear low-energy maximum of the $S$ factor shows up, and the logarithmic slope exceeds the $L_{C S}$ value by a large amount. The measured excitation function is overestimated by a standard Woods-Saxon CC calculation.
Our conclusion is that no hindrance effect is observed in ${ }^{58} \mathrm{Ni}+{ }^{64} \mathrm{Ni}$ in the measured energy range. This makes the trend of this system quite similar to ${ }^{40} \mathrm{Ca}+{ }^{96} \mathrm{Zr}$ at deep subbarrier energies and corroborates the recent suggestion that the availability of several states following transfer with $Q>0$, effectively counterbalances the Pauli repulsion that, in general, is predicted to reduce tunneling probability inside the Coulomb barrier.

\section{ACKNOWLEDGMENTS}

Valuable discussions with H. Esbensen are gratefully acknowledged. The XTU Tandem staff provided us with highquality beams and M. Loriggiola prepared targets of excellent quality. The research leading to these results received funding from the European Union Seventh Framework Program FP7/2007-2013 under Grant Agreement No. 262010 - ENSAR. P.C., S.S., and T.M. were partially supported by the Croatian Science Foundation under the project IP-2018-011257.
[1] M. Beckerman, M. Salomaa, A. Sperduto, H. Enge, J. Ball, A. DiRienzo, S. Gazes, Yan Chen, J. D. Molitoris, and Mao Nai-feng, Phys. Rev. Lett. 45, 1472 (1980).

[2] R. A. Broglia, C. H. Dasso, S. Landowne, and Å. Winther, Phys. Rev. C 27, 2433 (1983).

[3] D. Ackermann et al., Nucl. Phys. A 583, 129 (1995).

[4] G. Montagnoli and A. M. Stefanini, Eur. Phys. J. A 53, 169 (2017).

[5] C. L. Jiang et al., Phys. Rev. Lett. 89, 052701 (2002).

[6] C. L. Jiang, K. E. Rehm, R. V. F. Janssens, H. Esbensen, I. Ahmad, B. B. Back, P. Collon, C. N. Davids, J. P. Greene, D. J. Henderson, G. Mukherjee, R. C. Pardo, M. Paul, T. O. Pennington, D. Seweryniak, S. Sinha, and Z. Zhou, Phys. Rev. Lett. 93, 012701 (2004).

[7] S. Misicu and H. Esbensen, Phys. Rev. Lett. 96, 112701 (2006); Phys. Rev. C 75, 034606 (2007).

[8] H. Esbensen and S. Misicu, Phys. Rev. C 76, 054609 (2007).

[9] B. B. Back, H. Esbensen, C. L. Jiang, and K. E. Rehm, Rev. Mod. Phys. 86, 317 (2014).

[10] T. Ichikawa, K. Hagino, and A. Iwamoto, Phys. Rev. C 75, 057603 (2007).

[11] C. Simenel A. S. Umar, K. Godbey, M. Dasgupta, and D. J. Hinde, Phys. Rev. C 95, 031601(R) (2017).

[12] H. Esbensen and A. M. Stefanini, Phys. Rev. C 89, 044616 (2014).

[13] H. Timmers et al., Phys. Lett. B 399, 35 (1997); Nucl. Phys. A 633, 421 (1998).
[14] A. M. Stefanini et al., Phys. Lett. B 728, 639 (2014).

[15] H. Esbensen, G. Montagnoli, and A. M. Stefanini, Phys. Rev. C 93, 034609 (2016).

[16] G. Montagnoli, A. M. Stefanini, C. L. Jiang, K. Hagino, F. Galtarossa, G. Colucci, S. Bottoni, C. Broggini, A. Caciolli, P. Čolović, L. Corradi, S. Courtin, R. Depalo, E. Fioretto, G. Fruet, A. Gal, A. Goasduff, M. Heine, S. P. Hu, M. Kaur, T. Mijatović, M. Mazzocco, D. Montanari, F. Scarlassara, E. Strano, S. Szilner, and G. X. Zhang, Phys. Rev. C 97, 024610 (2018).

[17] A. M. Stefanini et al., Phys. Rev. Lett. 74, 864 (1995).

[18] K. Hagino, N. Rowley, and A. T. Kruppa, Comput. Phys. Commun. 123, 143 (1999).

[19] Ö. Akyüz and Å. Winther, in Nuclear Structure and Heavy-Ion Physics, Proceedings of the International School of Physics "Enrico Fermi," Course LXXVII, Varenna, edited by R. A. Broglia and R. A. Ricci (North Holland, Amsterdam, 1981).

[20] C. H. Dasso and G. Pollarolo, Phys. Lett. B 155, 223 (1985).

[21] C. L. Jiang, H. Esbensen, B. B. Back, R. V. F. Janssens, and K. E. Rehm, Phys. Rev. C 69, 014604 (2004).

[22] S. Raman, C. W. Nestor, Jr., and P. Tikkanen, At. Data Nucl. Data Tables 78, 1 (2001).

[23] T. Kibédi and R. H. Spear, At. Data Nucl. Data Tables 80, 35 (2002).

[24] Evaluated Nuclear Structure Data Files, National Nuclear Data Center, Brookhaven National Laboratory http://www.nndc.bnl. gov/. 